\title{
Using Daub achy Wavelet for Shot Boundary Detection
}

\author{
Ahmed Tariq, Nada Flail, Amaal Ghazi \\ Computer Sciences/University Of Technology/ Baghdad-Iraq
}

Abstract: SHOT Boundary Detection Is A Fundamental Step In Most Video Processing And Manipulation Applications. With The Wide Spread Of Video Usage Nowadays, The Rise For Automated Video Shot Boundary Detection Becomes An Essential. This Paper Summarises The Mostly Used Shot Boundary Detection Techniques, Then Suggests The Implementation Of Daubachy 4 Wavelet Along With Hsv Color Space In Order To Presents An Automated Shot Boundary Technique.

Keywords: Video Processing, Shot Boundary Detection, Daubechies Wavelet, Hsv

\section{Introduction}

Video Is The Most Effective Media For Capturing The World Around Us. By Combining Audio And Visual Effects, It Achieves A Very High Degree Of Reality. With The Widely Accepted Digital Video Standard And Low-Cost Hardware Support, Digital Video Has Gained Popularity In All Aspects Of Life. Video Plays An Important Role In Entertainment, Education, And Training. The Term "Video" Is Used Extensively In The Industry To Represent All Audiovisual Recording And Playback Technologies[1].

In Video Segmentation, A Video Sequence Is Segmented Into Physical Chunks Called Shots. By Definition, A Video Shot Is The Smallest Sequence Of Frames That Possesses A Simple And Self-Contained Concept. In Practice, Video Shots Are Identified And Segmented Using Scene Change Detection Techniques. A Change In Scene Will Mark The End Of The Previous Shot And The Start Of The Next One. Figure1 Shows The Way In Which A Video Sequence Is Segmented Into Shots[1].
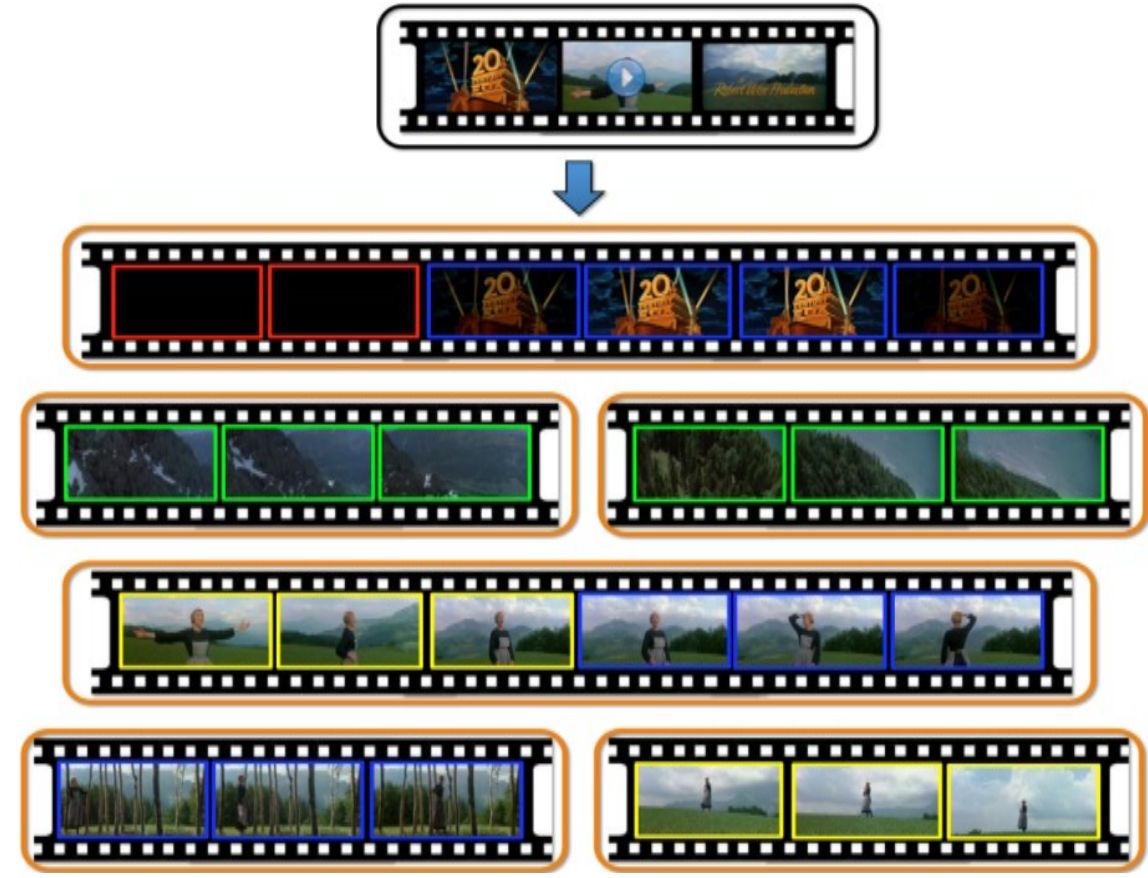

Figure 1:Segmentation And Representation Of Video Shots.

In The Segmentation Approach, A Video Shot Is An Atomic Unit That Can Be Manipulated For Representation And Retrieval. Figure1 Also Shows How A Video Shot Can Be Represented[1].

\section{Shot Detection}

The Success Of The Segmentation Approach Depends Largely On How Well The Video Materials Are Divided Into Segments Or Shots. This Involves Identifying Suitable Criteria To Decide Which Points In A Video Sequence Constitute Segment Boundaries. Manual Segmentation Has Been Done Effectively In The 
Movie Industry. However, Manual Segmentation Is Time Consuming And Prone To Error. Moreover, Its Results Are Biased Toward The Authors' Intents. With Advances In Image Processing Techniques, ComputerAided Segmentation Is Now Possible. The Main Objective Is To Detect The Joining Of Two Shots In The Video Sequence And Locate The Exact Position Of These Joins. These Joins Are Made By The Video Editing Process, And They Can Be Of The Following Types Based On The Techniques Involved In The Editing Process[1]:

\section{Abrupt Shot Transitions}

Which Are Also Known As Hard Cuts, Are Very Easy And Common To Detect Since The Two Frames That We Compare Are Unrelated. A Hard Cut Occurs In A Single Frame, Thus, If A Frame Contains Two Consecutive Shots $I 1$ And $I 2$ Then A Similarity Measure Computed In The Following Equation Is Used To Determine The Hard Cut By Comparing It Against A Certain Threshold[2].

$$
D\left(I_{1}, I_{2}\right)=\sum_{i=1}^{X} \sum_{i=1}^{Y}\left|I_{1}(i, j)-I_{2}(i, j)\right|
$$

Hard Cuts Are Reliable And Easy To Detect. A Hard Cut Generates A Temporal Visual Discontinuity In The Video Stream Presented By Isolated Peaks In The Feature Time Series. Current Hard Cut Detection Algorithms Are Different In The Features Used To Measure The Discontinuity And In The Schemes Used To Detect The Discontinuity. For Example, Some Use Shot Intensity Function And Pixel Spatial Distributions (I.E., The Position Of A Pixel As Well As Its Neighbors) For Their Features[2].

Most Work On Detecting A Sudden Scene Change Is Based On The Entire Images And Uses Different Metrics To Evaluate The Changes Between Successive Frames. The Most Common Schemes Are[2]:

\section{Pixel-Differences}

In This Technique, The Absolute Intensity Difference Between Two Corresponding Pixels Of Consecutive Frames Is Computed. This Number Is Compared Against A Threshold To Determine If A Shot Boundary Has Been Found.

\section{Likelihood Ratio}

This Algorithm Works On The Level Of Blocks Of Pixels Instead Of Pixels. In This Algorithm, The Frames Are Subdivided Into A Set Of Blocks, Which Are Then Compared On The Basis Of The Statistical Characteristics Of Their Intensity Levels.

\section{Histogram Comparison}

The Basic Idea In The Histogram Is That The Color Content Does Not Change Rapidly Within Shots. Thus, Hard Cuts And Other Short Lasting Transitions Can Be Detected As Single Peaks In The Time Series Of The Differences Between Color Histogram Of Contiguous Frames Or Of Frames At Certain Distance $K$ Apart.

\section{Motion Vector Counts}

Hard Cuts Are Also Accompanied By Motion Discontinuity. The Simplest Measure Of Motion Is The Pixel-Wise Frame Difference. At Hard Cut Locations, The Pixel-Wise Difference Is Usually Large. Unfortunately, This Simple Motion Measure Is Very Susceptible To Object And Global Camera Motion. Even If Global Camera Motion Is Compensated, Object Motion Still Poses A Significant Challenge To This Feature.

\section{Gradual Transitions}

As A Result Of The Increased Role Of Computer Technology In Video Production, Several Types Of Complex Gradual Scene Changes Have Begun To Appear In Video Clips. These Gradual Transitions Are Used To Enhance The Quality Of The Video Production. However, Gradual Transitions Are More Difficult To Detect Due To The Difficulty Of Modeling Of Gradual Shot Boundaries. Therefore, Comparison Based On Successive Frames Alone Is Not Adequate For The Detection Of Gradual Transitions. Gradual Transition Is An Important Class Of Effects And Includes Dissolving, Fading In, Fading Out, Mattes, And Translates. A Fade Is A Gradual Transition Between A Scene And A Constant Image (Fade Out) Or Between A Const Image And A Scene (Fade In). During A Fade, Images Have Their Intensities Multiplied By Some Value $A$. During A Fade In, $A$ Increase From 0 To 1, While During A Fade Out, $A$ Decrease From 1 To 0 . The Speed With Which $A$ Changes Controls The Fade Rate. A Dissolve Is A Gradual Transition From One Scene To Another, In Which The First Scene Fades Out And The Second Scene Fades In. Typically, Fade Out And Fade In Begin At The Same Time, And The Fade Rate Is Constant. Another Common Scene Break Is A Wipe, In Which A Line Moves Across The Screen, With The New Scene Appearing Behind The Line. The Presence Of Specific Effects Can Then Be Used As A Clue For Detecting Scene Changes As Opposed To Simple Shot Detection. A Matte Is A Progressive 
Obscuration Of Visual Field, Due To A Mask That Invades The Screen. A Translate Is A Spatial Edit: The First Shot Translates Out, Uncovering The Shot That Follows The Edit. Different Kinds Of Transitions Are Due To Panning And Zooming But These Are Not Usually Related To Shot Changes. Following Is A Survey Of The Common Algorithms That Have Been Proposed To Detect Gradual Transitions.

\section{Twin Comparisons}

This Scheme Takes Into Account The Cumulative Differences Between The Frames And Requires Two Cut-Off Thresholds: A Higher Threshold (Th) For Detecting Abrupt Transitions And A Lower One (Tl) For Gradual Transitions. In The First Stage The Higher Threshold Is Used For Detecting Abrupt Transitions. In The Next Stage The Lower Threshold Is Used And Any Frame For Which This Threshold Is Exceeded Is Declared To Be A Potential Transition Start Frame. However, For Most Gradual Transition, The Frame Differences Fall Below The Lower Threshold. Therefore, Such Transitions Cannot Be Detected Using The Twin-Comparison Technique.

\section{Plateau Detection}

Plateau Compares The Features Of The Of The $I$-Th Frame With That Of The $(N-K)$-Th Frame Instead Of Adjacent Ones. Then The Distance Measure Plotted As A Function Of Time Shows A Plateaulike Graph And Plateau Detection Is Performed To Detect The Shot Boundaries. In This Algorithm, Abrupt Changes And Gradual Transitions Are Detected Separately. An Abrupt Scene Change Is Declared If There Is A Sharp Peak In The $D i$ Plot, Where $D i$ Is Simply The Difference Between Frame $I$ And Frame $I+1$.

\section{Proposed Shot Boundary Detection}

Several Features Have Been Used In This Proposal, Which Are:

\section{HSV Color System Feature}

Color Is The Brains Reaction To A Specific Visual Stimulus. A Color Space, Also Known As A 'Color Model', Is A System For Describing Color Numerically. With The Help Of Color Spaces, Color Can Be Specified, Created And Visualized, Either Between People Or Between Machines Or Programs.[3]

The HSV (Hue, Saturation, And Value), Also Known As HSB (Hue, Saturation, And Brightness) Color Space Was Created In 1978 By Alvy Ray Smith. It Is Quite Similar To The Way In Which Humans Perceive Color. The Colors Used In HSV Model Can Be Defined Clearly By Human Perception, Which Is Not Always The Case With RGB. The HSV Color Space Is A Basic Color Space In Some Image-Processing Software Like Photoshop.[4].

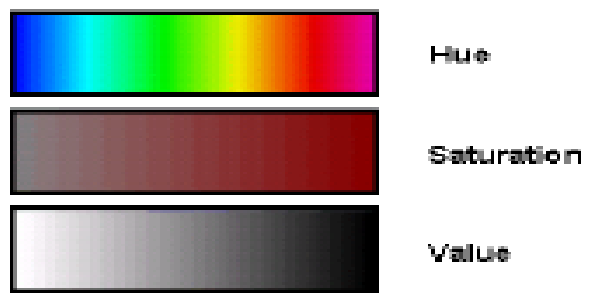

Figure 2: Perceptive Measures In HSV Color Model

\section{Daubachy 4 Wavelet Texture Feature}

A Wavelet Transform Is Equivalent To A Filtering Process With Two Filters That Are Quadrature Mirror Filters And Divide The Time Series Into A Wavelet Part, Which Represents The Detail, And Another Smoother Part[5].

Daubechies(1988) Discovered An Important And Useful Class Of Such Filter Coefficients. The Simplest Set Has Only 4 Coefficients (DAUB4), And Will Serve As A Useful Illustration. Consider The Following Transformation Acting On A Data Vector To Its Right[5]. 


$$
\left[\begin{array}{cccccccccccc}
c_{0} & c_{1} & c_{2} & c_{3} & & & & & & & \\
c_{3} & -c_{2} & c_{1} & -c_{0} & & & & & & & & \\
& & c_{0} & c_{1} & c_{2} & c_{3} & & & & & & \\
& & c_{3} & -c_{2} & c_{1} & -c_{0} & & \bullet & \bullet & & & \\
& & & & & \bullet & \bullet & c_{0} & c_{1} & c_{2} & c_{3} \\
& & & & & & & & c_{3} & -c_{2} & c_{1} & -c_{0} \\
c_{2} & c_{3} & & & & & & & & c_{0} & c_{1} \\
c_{1} & -c_{0} & & & & & & & & & & \\
& & & & & & & & & c_{3} & -c_{2}
\end{array}\right]
$$

Figure 3: Cut Transition At Frame 121

The Matrix Is Arranged In Such A Way That Cyclic Continuity Of The Data Is Assumed, Much As In Fourier Analysis. The Action Of This Matrix Is To Perform Two Convolutions With Different, But Related, Filters, $C 0, C 1, C 2, C 3$ ( ) $=H$ And $C 3,-C 2, C 1,-C 0(\mathrm{)}=G$, Each Resulting Time Series Of Filtered Data Points Is Then Decimated By Half, So That Only Half As Many Data Points Remain, Then Both Filtered Time Series, Thus Decimated, Are Interleaved. Think Of $H$ As The Smoothing Filter And $G$ As The Wavelet Filter. They Produce The Smooth And Detail Information, Respectively[5].

\section{Statistical Monents Features}

Statistical Features Are Extracted For Each Vector By Using The Following Equations:

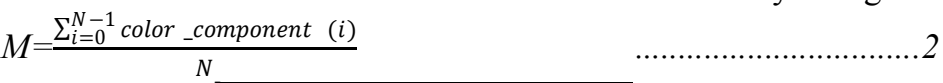

$$
\begin{aligned}
& \text { STD }
\end{aligned}
$$

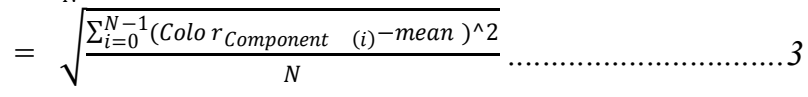

\section{Implementation \& Results}

This Work Concerns Upon Testing And Evaluating Multilevel Scheme Which Relies On Daub4 In HSV Color Space As A Level Of These Features (Daub4 Represents Texture Feature) And On Other Features As Building Stones In Investigation. The Following Results Are Subsamples Of Applying The Previously Mentioned Features (Mean, STD) To Each Of Daub4 Sub-Band (LL, LH, HL, HH) Separately.

Applying Mean And Standard Devotion On Each Daub4 Sub-Band Returns Similar Results For (LL, HL, And HH) Against Each HSV Color-Bands With The Exaction Of Applying Mean On LH Daub4 Sub-Band Aganist Hue Color Component Of HSV Color System.

A Cut Transition Shot Can Be Directly Found By Using Mean On Hue-LH Daub4 Sub-Band. Figure 3 Shows A Chart For Its Value While Figure 4 Shows The Transition In Frames.

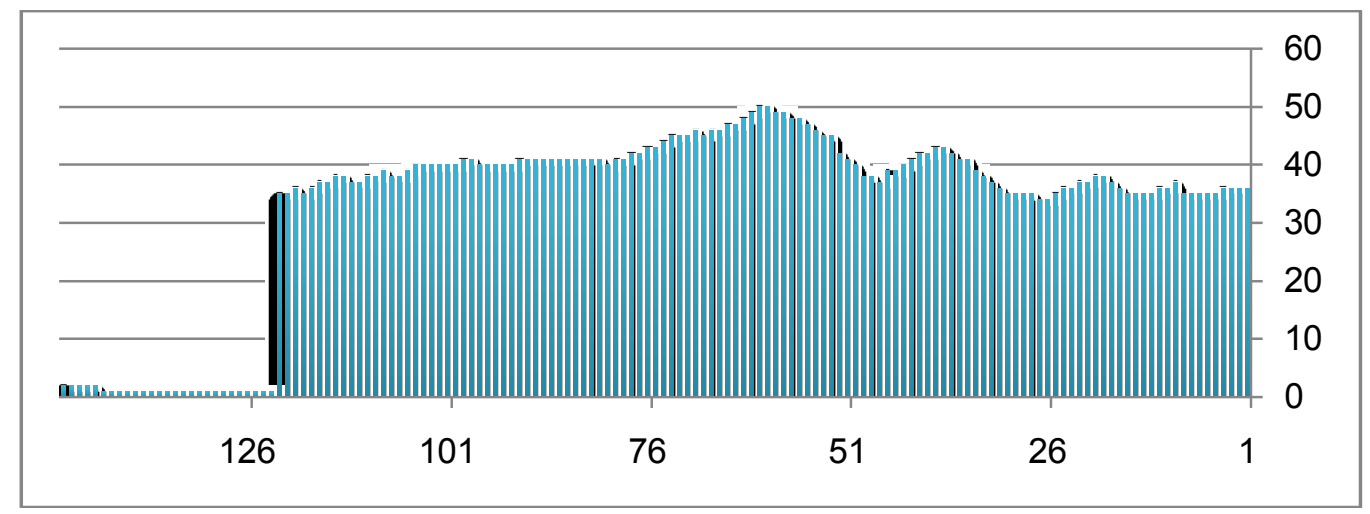

Figure 4: Frame Sequence 120-124 With Cut Transition Between 121 And 122

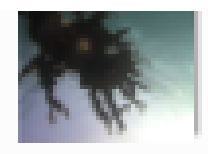

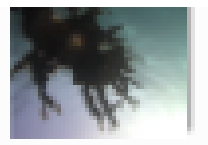

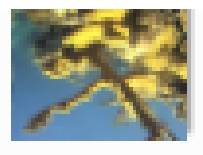

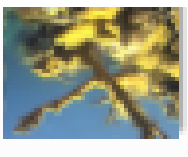

Figure 5: Gradual Transition Between Frames 53-57 And Frames 86-92 
A Gradual Transition Shot Can Be Found By Using Mean On Hue-LH Daub4 Sub-Band., Where Transitions Can Be Found By Symmetric Decrease-Zero Value-Increase In The Previously Mentioned Sub Band. Figure 5 Shows A Chart For Its Value While Figure 6 And 7 Shos The Transition In Frames.

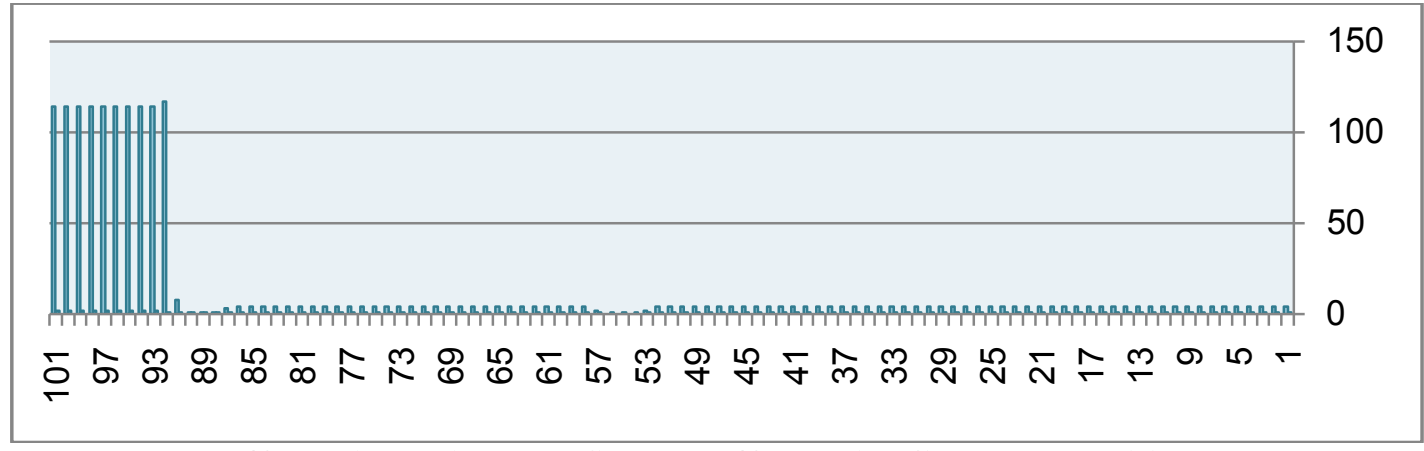

48-57 Figure 6: Frame Sequence 48-57 With Gradual Transition
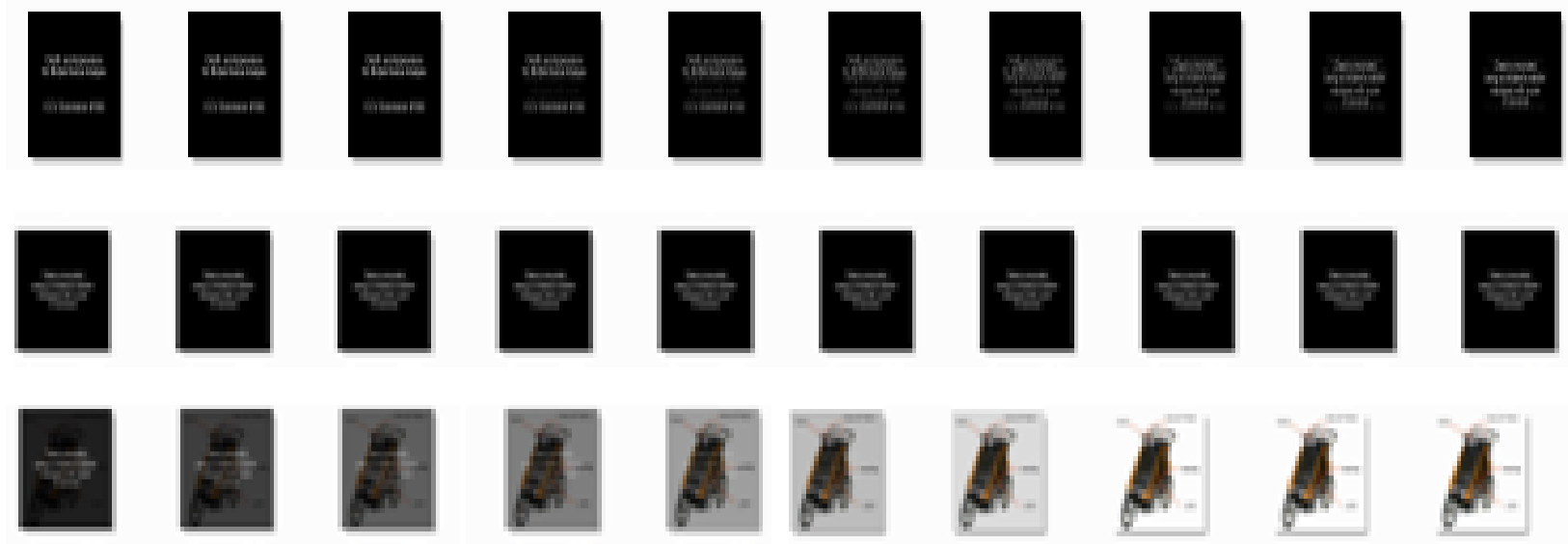

Figure 7: Frame Sequence 75-94 With Gradual Transition

\section{Conclusions \& Future Work}

Implementation Of Daubachy 4 Wavelet Along With HSV Color Space Can Provide An Automated Shot Boundary Technique Which Can Be Very Helpful As An Pre-Processing In Many Video Processing And Key Frame Detection Applications. Yet Wavelets Severe From Expensive Computations Both In Time And Processing, Thus In Order For This Work To Be Applicable In A Useful Application These Aspects Need To Be Taken Under Consideration.

\section{References}

[1]. Yi Zhang And Tat-Seng Chua, " Video Modeling And Retrieval", Multimedia Image And Video Processing, CRC Press, 2001.

[2]. Eyas EL-QAWASMEH," Scene Change Detection Schemes For Video Indexing In Uncompressed Domain", INFORMATICA, 2003, Vol. 14, No. 1, 19-36.

[3]. Farag \& Abdel-Wahab," Video Shot Boundary Detection", Idea Group Inc., 2005.

[4]. Haiyan Xie, " Key Frame Segmentation In Video Sequences- Applied To Reconstruction Of 3D Scene", M.SC. Thesis, Department Of Technology, University Of Kalmar, Sweden, 2008.

[5]. Hartmann, "Wavelets", ATMS 552 Notes, P 255-275, 2014 